\title{
Comparison on the Biochemical Characteristics between Alfalfa Sterile Line and Maintainer Line
}

\author{
$Y A N$ Dong $^{1}, \quad X U$ Xiaobo $^{1}, \quad G U O$ Qiang $^{1}$, and $X U \mathrm{Bo}^{1, *}$ \\ ${ }^{1}$ College of Animal Science and Technology, Jilin Agricultural University, Changchun 130118, \\ China
}

\begin{abstract}
To study on the morphological character of cytology and physiological and biochemical mechanism of alfalfa cytoplasmic male sterile line, this paper took sterile line MS-GN1A and maintainer line MSGN1B as raw material, separately conducted microscopic observation to anther in different development stage and determined the content of soluble sugar, starch, soluble protein, free proline and malondialdehyde, and the activity of antioxidant enzyme (catalase, peroxidase and superoxide dismutase). The results showed that: 1. Compared with maintainer line, the pollen grain of alfalfa cytoplasmic male sterile line presented shriveled appearance, smaller size with deletion and few; during the tetrad period of pollen, tapetum cell abnormally grew; callose and epidermis didn't disintegrate and couldn't release pollen grain; pollen abortion of alfalfa cytoplasmic sterile line was related to the abnormal development of connective vascular bundle. 2. With the growth and development of bud and the development and maturity of pollen, the content of soluble sugar, starch, soluble protein, and free proline showed deficient phenomenon in each development period of bud of sterile line MS-GN1A; as peroxidation index of membranous substance, the activity of malondialdehyde and antioxidant enzyme was higher than it of homotype maintainer line MS-GN1B.
\end{abstract}

\section{Introduction}

The acquisition of male sterile lines is of great significance for the utilization of plant heterosis. According to the male sterile genotypes, they can be divided into three types, namely, the nuclear sterile type, the cytoplasmic sterile type and the nuclear cytoplasmic interactive sterile type.The flower development of plants needs to go through a series of complex processes, which may directly or indirectly affect the occurrence of sterility and abortion.A large number of studies have shown that male sterility in plants is related to physiological and biochemical processes such as gene expression and regulation [1], material metabolism [2], energy metabolism [3], membrane stability [4] and photosynthesis [5].However, there are few studies on cytoplasmic male sterility in Medicago sativa L. the physiological and biochemical mechanism of cytoplasmic male sterility in Medicago sativa L. is not clear.In this experiment, the anthers of CMS line and its maintainer line were used

*Corresponding author: xubo0308@126.com 
as experimental materials to observe the microstructure.To explore the critical period and occurrence mode of pollen abortion of male sterile plants of Alfalfa, and the changes and connections of the wall tissue and cell microstructure of traditional Chinese medicine during the abortion process; to analyze the content of soluble sugar, starch, soluble protein, free proline, malondialdehyde and the activity of catalase and other enzymes, so as to explore the differences between cytoplasmic male sterility and fertility of Alfalfa.The purpose of this study is to reveal the physiological and biochemical mechanism of cytoplasmic sterility and abortion of Alfalfa, and to provide theoretical basis for further understanding of the mechanism of sterility of Alfalfa.

\section{Materials and Methods}

\subsection{Test materials}

The female parent material came from 2008. Researcher Xu Ankai, Jilin Academy of Agricultural Sciences, used the satellite technology to find the male sterile material MS-GN [6]. The male parent material is a good variety of Alfalfa in the United States, Russia and China with moderate growth period, compact plant type and strong resistance. Careful field observation and fertility identification were carried out to select complete male sterility and agronomic characters. The lines with good economic characters were used as test parents.All the materials were from Jilin Academy of Agricultural Sciences.

\subsection{Preparation and observation of microstructure of anther paraffin section}

Five plants of alfalfa sterile MS-GN1A and its maintainer MS-GN1B were selected respectively. One branch was selected from the top, middle and bottom of each plant, and ten flower buds were marked respectively. The flower buds were taken at budding stage, and the flower buds at different development stages were collected at flowering stage. According to the morphological characteristics of the flower buds, combined with microscopic examination results and development time, they were divided into stage I (budding stage $24 \mathrm{~h}$ ) and stage II (budding stage $40 \mathrm{~h}$ ). There are five stages of development: stage III $(60 \mathrm{H}$ in budding stage), stage IV $(68 \mathrm{H}$ in budding stage) and stage V ( $84 \mathrm{~h}$ in budding stage).In terms of cytology, referring to the flower bud classification of these five stages, the anthers were taken out, paraffin embedded sections were made, and the anthers at different stages of development were observed by Nikon-E200 optical microscope.

\subsection{Test methods of related physiological and biochemical indexes}

In this experiment, 2 grams of anthers at the above 5 different development stages were respectively taken and frozen in liquid nitrogen, and then stored at $-80{ }^{\circ} \mathrm{C}$ for future use. Each experiment was repeated for three times.Anthrone colorimetry, Coomassie brilliant blue G-250 method, sulfosalicylic acid method, TBA method, ultraviolet absorption method, guaiacol method and NBT method were used to determine the content of soluble carbohydrate (soluble sugar and starch), soluble protein, free proline, malondialdehyde and the activity of catalase, peroxidase and superoxide dismutase.Some of the test methods were adjusted slightly and repeated 3 times. 


\subsection{Data processing and analysis}

Collect and record the experimental data, and use 2016 version Excel to sort out and analyze the data.

\section{Results and analysis}

\subsection{Observation and analysis of anther cytology of cytoplasmic male sterile line and maintainer line}

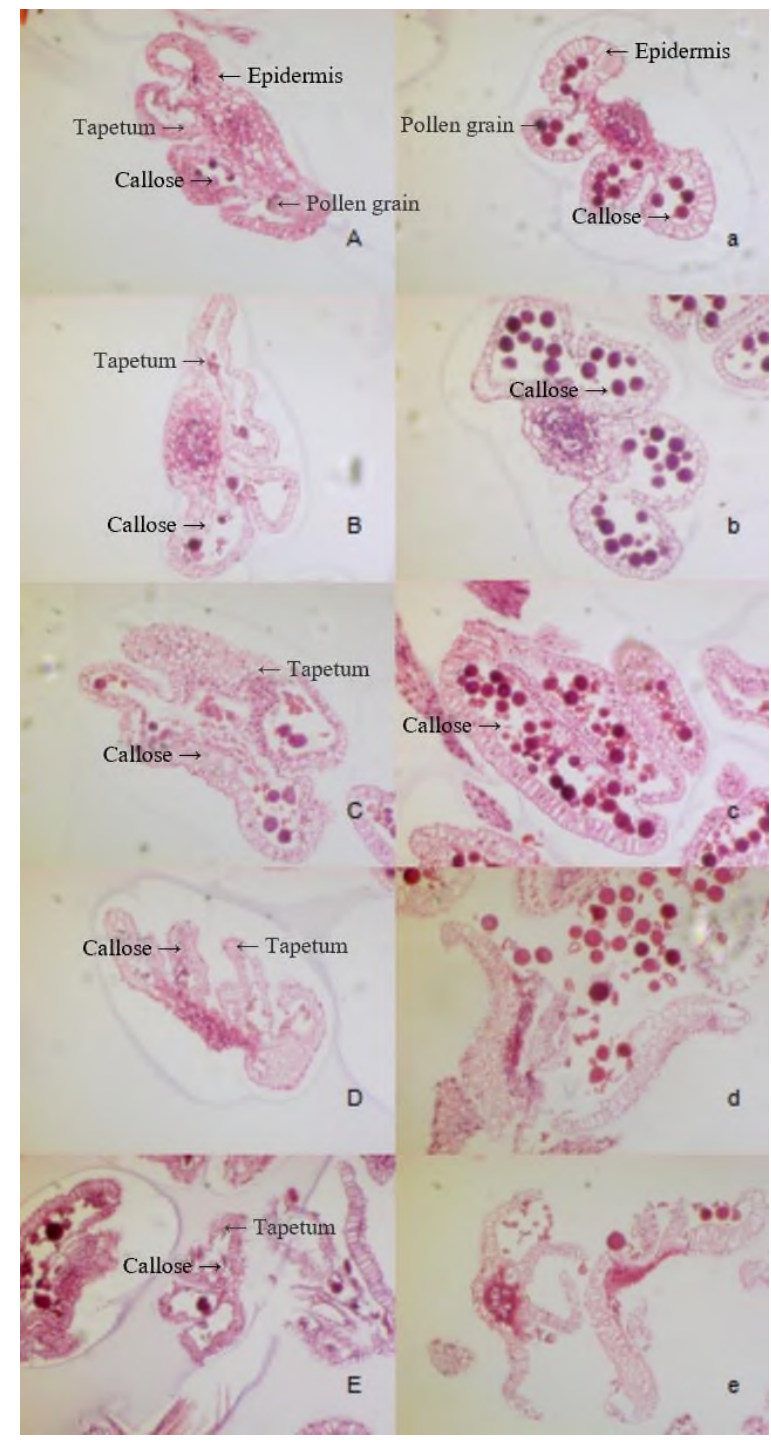

Fig.1. Microscope detection of five periods of anther section. in cytoplasmic male sterile line MSGN1A and maintainer MS-GN1B. Note: The anther sections of CMS lines with five periods of capital letters on the left and those with small letters on the right are the anther sections of maintainer lines in the same period. 
As shown in Fig. 1, from stage I to V, the spore grains of the maintainer line are plump and in good shape, and the microspores of the cytoplasmic male sterile line are shrunken and relatively small with defects (Fig. A, Fig. B, Fig. C, Fig. D and Fig. E).It was found that the tapetum disintegration of the maintainer line in stage I had no trace (Fig. a), while that of the cytoplasmic male sterile line from stage I to stage $\mathrm{V}$ was still clear (Fig. A).In stage I and II, the development of vascular bundle of anther septum of CMS line and maintainer line was basically consistent (Fig. A, Fig. B, Fig. a and Fig. b).In stage III and IV, the vascular bundle of anther septum of cytoplasmic male sterile line was clearly seen (Fig. C and Fig. D), and the vascular bundle of anther septum of maintainer line began to disintegrate and microspore had mitosis (Fig. c and Fig. d).From stage IV to V, the epidermis and callose of maintainer line disintegrated, microspore mitosis released mature pollen grains (Fig. e), the epidermis and callose of cytoplasmic male sterile line did not disintegrate, and microspore did not undergo mitosis to release anthers (Fig. E).However, there was no significant difference between the sterile line and the tetrad stage.

\subsection{Analysis of physiological and biochemical indexes of cytoplasmic male sterile line and maintainer line}

\subsubsection{Comparative analysis of soluble sugar content}

It can be seen from Fig. 2 that the trend of change of soluble sugar content of anthers of CMS lines and Their Maintainers in different development periods is the same as a whole. The change range of cytoplasmic male sterile lines from stage IV to stage $\mathrm{V}$ is relatively large, and the maximum change range of maintainer lines is from stage III to stage v. the highest value of soluble sugar content of CMS lines is more than the lowest value of soluble sugar content of maintainer lines.D

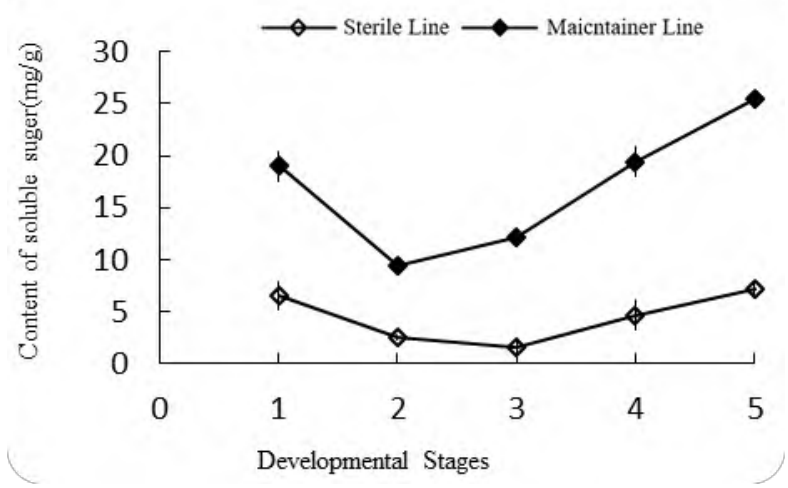

Fig.2. Soluble sugar content of different developmental stages of anther on MS-GN1A and MSGN1B.

\subsubsection{Comparative analysis of starch content}

With the development of flowers, the change of starch content in the anthers of CMS plants of Alfalfa is not very obvious. As can be seen from Figure 3, the change from phase I to phase $\mathrm{V}$ is very small, with rise and fall, and the last phase $\mathrm{V}$ content is slightly lower than that of phase I.The starch content in flower organs Cof maintainer lines increased gradually. The highest starch content was in stage $\mathrm{V}$, which was more than 1.5 times of that in stage I. 
the highest starch content of cytoplasmic male sterile lines was close to that in stage I of maintainer lines.

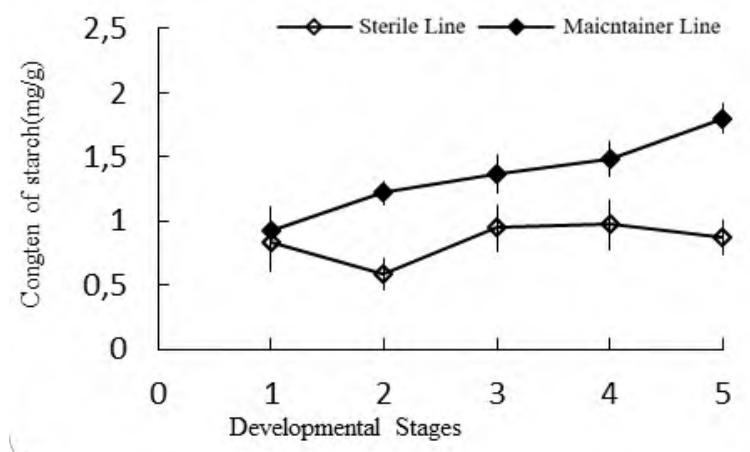

Fig.3. Starch content of different developmental stages of anther on MS-GN1A and MS-GN1B.

\subsubsection{Comparative analysis of soluble protein content}

In the process of flower development, the soluble protein content of both male sterile line and maintainer line showed a downward trend. The soluble protein content of the first stage male sterile line was slightly lower than that of maintainer line, and then the difference of soluble protein content in each stage was increasing.From stage IV to stage V, the decrease of soluble protein content of CMS line was the most significant, while that of maintainer line was stable from stage I to stage $\mathrm{V}$.The ratio of the soluble protein content in the floral organs of the male sterile line between stage $\mathrm{V}$ and stage I was about 0.6 , and that of the maintainer line was about 0.75 .

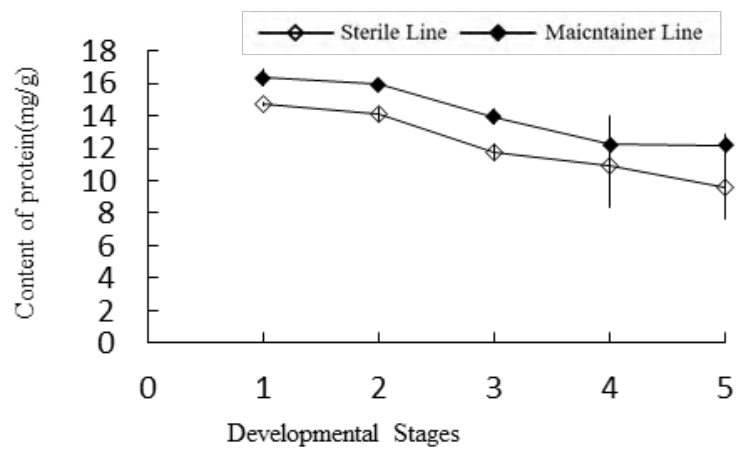

Fig.4. Soluble protein content of different developmental stages of anther on MS-GN1A and MSGN1B.

\subsubsection{Comparative analysis of free proline content}

Free amino acids play an important role in microspore development, and proline is an essential substance in pollen metabolism [7-8].It can be seen from Fig. 5 that the content of free proline in CMS lines decreased from stage I to stage IV, and increased sharply from stage IV to stage $\mathrm{V}$. the content of free proline in stage $\mathrm{V}$ was 3.5 times higher than that in 
stage IV.The content of free proline in maintainer line showed a trend of constant decline. As a whole, the content of free proline in stage $\mathrm{V}$ was significantly higher than that in maintainer line.

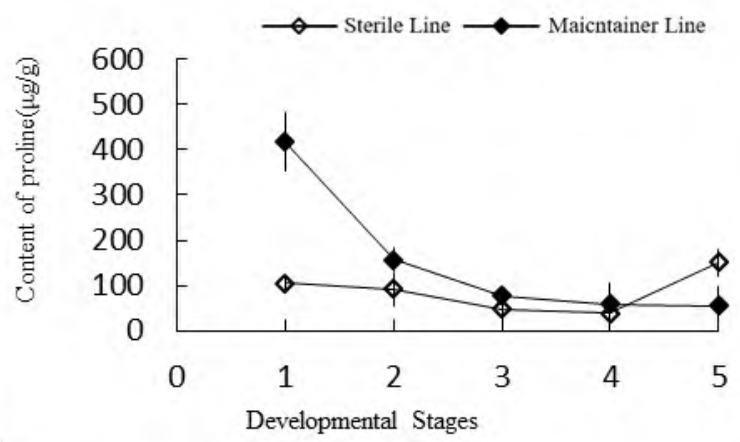

Fig.5. Proline content of different developmental stages of anther on MS-GN1A and MS-GN1B.

\subsubsection{Comparative analysis of malondialdehyde content}

An important indicator of the degree of membrane oxidation is the content of malondialdehyde (MDA) ${ }^{[9]}$. The test results are shown in Fig. 6. The MDA content of CMS line I and maintainer line is basically the same, and the MDA content of maintainer line III is about three times of that of CMS line.For the five stages of flower development, except that the MDA content of CMS line in stage IV was higher than that of CMS line, the MDA content of CMS line in other stages was higher than that of CMS line, especially in stage III.

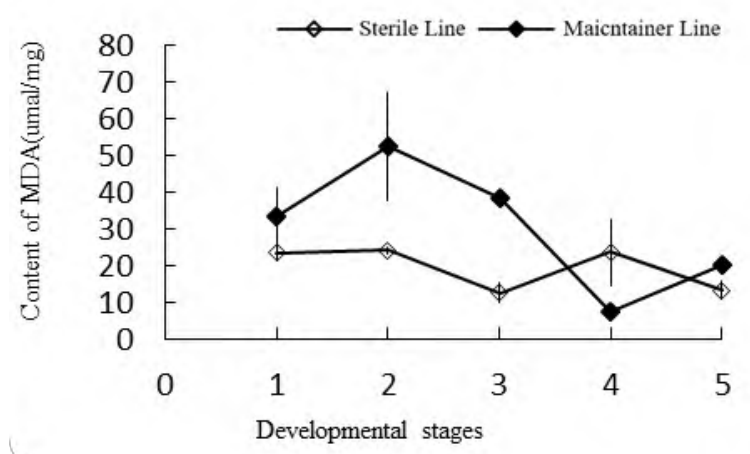

Fig.6. MDA content of different developmental stages of anther on MS-GN1A and MS-GN1B.

\subsubsection{Catalase activity comparison}

It can be seen from Figure 7 that the detection results show that the cat activity of the sterile line has just begun to decline. As the peanut grows, its activity gradually increases, and the cat activity of the maintainer line as a whole shows an upward trend, but the cat activity of the maintainer line is lower than that of the sterile line, and it is always kept at a low level.In the whole period of flower development, the cat activity of sterile line was always 
greater than that of its maintainer line, and the difference of CAT activity value in stage IV was the most obvious.

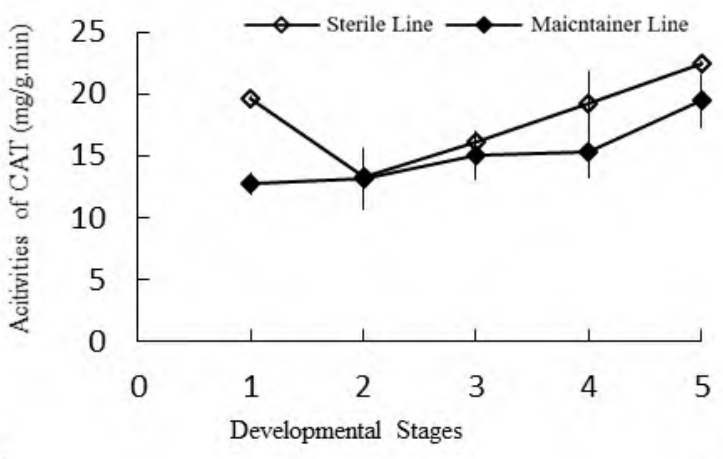

Fig.7. CAT activities of different developmental stages of anther on MS-GN1A and MS-GN1B.

\subsubsection{Comparison of peroxidase activity}

It can be seen from Fig. 8 that POD activity of sterile line is higher than that of maintainer line in all stages of Alfalfa flower development, and the difference in stage IV is the most obvious.For cytoplasmic male sterile lines, POD activity decreased before the third stage, decreased significantly from the first stage to the second stage, and there was no significant difference between the second stage and the third stage. POD activity increased significantly in the fourth stage, which may be the protective response of reactive oxygen stress. POD activity in the fifth stage was similar to that in the third stage.The POD activity of the maintainer line decreased as a whole, especially from stage I to stage II, with little change from stage II to stage $\mathrm{V}$.

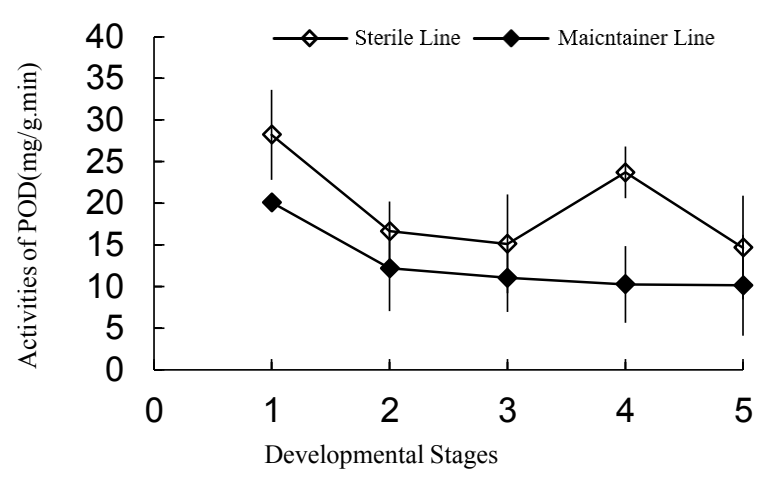

Fig.8. POD activities of different developmental stages of anther on MS-GN1A and MS-GN1B.

\subsubsection{Activity comparison of superoxide dismutase}

The physiological mechanism of Male Abortion shows that the metabolism of free radicals is closely related to the abortion of pollen in anthers [10].As an important antioxidant enzyme, superoxide dismutase (SOD) plays an important role in protecting the body from free radicals [11].As shown in Figure 9, the overall trend of SOD activity of sterile line and 
its maintainer line showed a decreasing trend. However, the activity of SOD in CMS line was significantly higher than that in maintainer line.

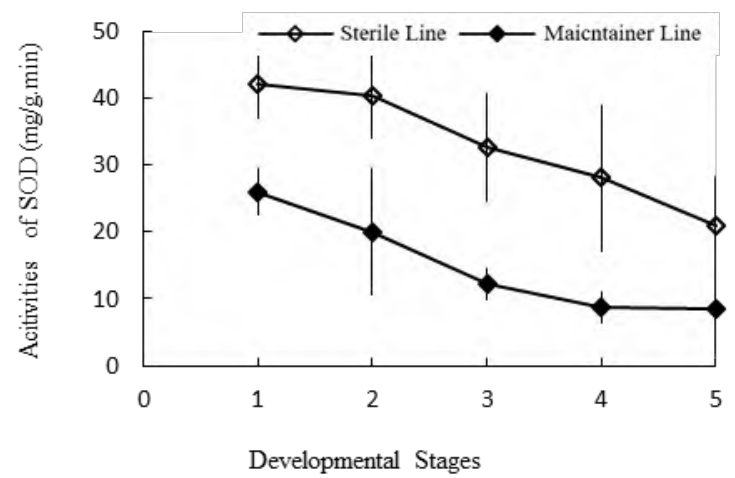

Fig.9. SOD activities of different developmental stages of anther on MS-GN1A and MS-GN1B.

\section{Discussion}

\subsection{Study on the cytological microstructure of the anther of cytoplasmic male sterile line and its maintainer line of Alfalfa}

For male sterility of different kinds of plants, many studies have shown that abnormal tapetum development is one of the important reasons for plant abortion, and the key reason for nuclear male sterility of alfalfa is also the abnormal tapetum development [12].In this experiment, the tapetum cells of pollen in the tetrad stage developed abnormally into the abortion stage of cytoplasmic male sterility of alfalfa.In the early stage of microspore development, the tapetum cells of the male sterile line were maintained to disintegrate and supply nutrients to the spores, while the tapetum of the male sterile line was abnormal and could not form normal microspores, and the pollen grains were shriveled, missing and few.

The vascular bundle of drug septum is a channel for plants to absorb water and transport nutrients to the anther chamber for pollen formation and development [13].The main reason is that the metabolism of the drug septum vascular bundle and the drug chamber is disordered during the normal development of microspore, which results in the loss of nutrients in the spore. In addition, it was also found that the callose and epidermis of the cytoplasmic male sterile line of Alfalfa did not disintegrate, which made the original quantity of pollen grains less and the poor quality could not be released, so the sterile effect was obvious.

\subsection{Relationship between nutrient metabolism of cytoplasmic male sterile line and its maintainer line of Alfalfa}

In the nineties of the last century, domestic scholars proposed the theory of "material deficiency" on the nutrient metabolism of CMS lines in corn [14], which showed that microspore development abnormality was directly related to organ structure level, nutrient and energy deficit, and some studies showed that the change of proline content was in direct proportion to pollen fertility [15]. The proline content of the first four developmental stages of the maintainer was higher than that of the male sterile line, and the proline content of the last stage was higher than that of the maintainer line.The lack of proline and the sudden increase of proline content in the later stage of flower bud development may affect 
the changes of metabolism structure, which may lead to the occurrence of microspore sterility and abortion.

Soluble protein is the main component of protoplasts and plays an important role in the growth and development of plant anthers and energy metabolism [16].This study showed that the soluble protein content of sterile lines at all stages was significantly lower than that of maintainer lines, which was consistent with the results of carrots [17] and other plants, but quite different from those of cabbage [18] and pepper [19]. Soluble protein deficiency is the key factor leading to pollen abortion of alfalfa.

The change of soluble carbohydrate metabolism has a very important influence on the material formation and energy metabolism in the process of Anther Development [20]. The content of the maintainer line was higher than that of the sterile line in every period, which indicated that carbohydrate deficiency affected the Microsporogenesis and resulted in pollen sterility or abortion in the process of anther development.

\subsection{The relationship between cytoplasmic male sterile line of Alfalfa and its membrane stability}

Membrane stability is the basic condition to maintain the normal life activities of cells. In the process of using oxygen, plants will accumulate active oxygen in the body, which will destroy membrane stability, leading to physiological and biochemical disorders [21].It was found that in stage IV, the male sterile line with MDA content and cytoplasmic male content exceeded the maintainer line, and the stability of membrane was poor.It is speculated that the reason of sterility may be due to the change of lipid membrane permeability of anther during the fourth stage of anther powder dispersion.

Enzymes are essential substances in the physiological and biochemical processes of all plants.Pod is an enzyme that can oxidize free radical indoleacetic acid and catalyze various reactions. Cat and SOD can clearly metabolize excess aerobic free radicals to keep the active oxygen in a balanced state [22].In this experiment, the difference between peroxidase and maintainer of stage IV male sterile line was the largest, which was the same as the change period of malondialdehyde.It can be seen that the fourth stage of anther dispersal is the key development stage of abortion. However, for the very complex mechanism of male sterility, it is not enough to explore the cause of sterility only from the physiological and biochemical mechanism, but also need to use the methods of cytology and molecular biology to conduct in-depth research and exploration.

\section{Conclusions}

\subsection{Cytological characteristics of cytoplasmic male sterile anthers and microspores of Alfalfa}

The early stage of microspore development, keep the system supply nutrients to the spore disintegration of tapetal cells, cytoplasmic male sterile lines tapetal abnormal could not form normal microspore, maintainer of the pollen grain, granular size, number, cytoplasmic male sterile lines pollen grains present a dry state, and smaller and there are missing, the number of less. The vascular bundle of the maintainer line was basically completed with the decline and disintegration of the tetrad, but the morphology and structure of the vascular bundle of the cytoplasmic male sterile line were clear and not significantly different from that of the tetrad period. The main reason was that the disruption of the vascular bundle of the diaphragm and the metabolism of the drug compartment affected the development of microspores.In addition, it was found that the cytoplasmic male sterile line callose and 
epidermis did not disintegrate in alfalfa, so that the pollen grains with low quantity and poor quality could not be released and the sterility effect was obvious.

\subsection{Physiological and biochemical characteristics related to cytoplasmic male sterility of Alfalfa}

With the growth and development of flowers and pollen, the content of soluble sugar, starch, soluble protein and free proline of MS-GN1A in the cytoplasmic male sterile line of alfalfa showed deficiency at each stage of anther development, while the activity of malondialdehyde and antioxidant enzyme, as indicators of membrane peroxidation, was higher than that of MS-GN1B.Compared with the cytoplasmic male sterile line and its maintainer line of alfalfa, the deficiency of nutrients is the main cause of male sterile and abortion during flower development.The abnormal membrane stability of the cytoplasmic male sterile anther of alfalfa resulted from the protective reaction of the membrane.And through the study found that the cytoplasm male sterile line and maintainer of the membranous stability abnormalities in IV period is most significantly, thus it can be seen, the anther powder IV period is the key cause abortive, but for the complicated mechanism of male sterility, only from the physiological and biochemical mechanism to explore the causes of infertility is not enough, still need to use cytology and molecular biology methods such as in-depth study and explore.

\section{Reference}

1. K. Suzuki, N. Aoki, H. Matsumura, et al. Cooling water before panicle initiation increases chilling in-duced male sterility and disables chilling induced expression ofgenes encoding OsFKBP 65 and heat shock proteins in ricespikelets. Plant Cell Environ, 7, 21-36 (2015)

2. L. Chen, Y. Liu, Male sterility and feritility resto-ration in crops, Annual Reviewof Plant Bialogy, 33(3), 123-148 (2014)

3. W. Yue, Z. Yanxia, L. Yanli, et al. Study on the difference of anther protein between wheat ven type cytoplasmic sterile plants and fertile plants, Journal of Northwest botanical Sciences, 41 (5), 1135-1145 (2016)

4. H. Xiaoyu. Physiological and biochemical analysis of radish male sterile line and maintainer line , Shandong Agricultural Science, 44 (12), 36-38 (2012)

5. Z. Yaowen. Photosynthetic characteristics of Isonuclear male sterile lines and Their Maintainers in Brassica napus, Chinese Journal of oil crops, 5 (3), 49-55. (2012)

6. X. Ankai, J. Rui, Y. Hongzhu. Research progress in application of Alfalfa male sterile lines, Grassland and animal husbandry, (6), 56-59 (2013)

7. H. Haiyan, W. Yu, Y. Qinghua, et al. Changes of enzyme activity in leaves of different resistant alfalfa plants infected with pseudodiscus, Chinese Journal of Grassland Science, 37(3),55-59 (2015)

8. F. Xiaolei. Research progress in physiology and biochemistry of plant male sterility Crop journal, 4(3),32-38 (2014)

9. Y. Fengyan, S. Fengling, G. Cuiping, et al. Comparison of physiological and biochemical characteristics between male sterile and fertile alfalfa plants, Chinese Journal of Grassland Science, 06, 60-65. (2014)

10. W. Junsheng. Analysis of active oxygen metabolism and gene expression in anther of wheat physiological male sterility. Xi'an: Northwest University of agriculture and 
forestry science and technology (2010)

11. H. Xiaoyu. Physiological and biochemical analysis of radish male sterile line and maintainer line, Shandong Agricultural Science, 44(12):36-38 (2012)

12. K. Jungen. Anther Abortion characteristics and gene expression profile analysis of four types of male sterile lines in cabbage. Ph.D. thesis, Chinese Academy of Agricultural Sciences. (2006)

13. H. Jinguo, Li Zebing. Preliminary study on the inheritance of sterility in four cytoplasmic male sterile lines of rice, Journal of Huazhong Agricultural University, (1985)

14. F. Xiaolei. Research progress in physiology and biochemistry of plant male sterility, Crop journal, 4 (3), 32-38 (2012)

15. Y. miaobao, Y. Zhilong, L. Taosheng, Genetic principles of cytoplasmic male sterility in plants, Beijing: Agricultural Press, (1980)

16. L. Jing, C. Yajun, Z. Lu, et al., Effect of water nitrogen interaction on physiology, biochemistry and turf quality of Poa pratensis, Chinese Journal of Grassland Science, 04, 42-48 (2016)

17. L. Yanrong, H. Xiaohong, Wang Yong, et al., Physiological and biochemical characteristics of carrot male sterile lines, Journal of North China agriculture, 12 (2): 28-33 (2004)

18. S. Rifei, F. Zhiyuan, Z. Shujiang, et al., Biochemical analysis of cytoplasmic male sterile line of Chinese Cabbage, Journal of horticulture, 27 (3), 187-192 (2007)

19. G. Sansheng, M. Aijun. Biochemical characteristics of male sterile anthers in Pepper Beijing Agricultural Science, 15 (2), 26-27 (1997)

20. Z. Lili, W. Jiahai, Chen Ying, et al., Effect of drought on physiological characteristics of Tall Fescue and evaluation of drought resistance, Chinese Journal of Grassland Science 04, 15-20 (2015)

21. M. K. Sledge, I. M. Ray, G. Jiang, An expressed sequence tag SSR map of tetraploid alfalfa ( Medicago sativa, L.), Theoretical and Applied Genetics, 111(5), 980-992 (2005)

22. H. Shoulin, G. Mingde, W. Hanquan, et al., Analysis of nutritional value of different alfalfa varieties, Soil and water conservation research, 12 (4), 217-219 (2005) 